\title{
Negative Energy Densities in Quantum Field Theory
}

\author{
L.H. Ford \\ Institute of Cosmology \\ Department of Physics and Astronomy \\ Tufts University, Medford, MA 02155USA *E-mail: ford@cosmos.phy.tufts.edu
}

\begin{abstract}
Quantum field theory allows for the suppression of vacuum fluctuations, leading to sub-vacuum phenomena. One of these is the appearance of local negative energy density. Selected aspects of negative energy will be reviewed, including the quantum inequalities which limit its magnitude and duration. However, these inequalities allow the possibility that negative energy and related effects might be observable. Some recent proposals for experiments to search for sub-vacuum phenomena will be discussed. Fluctuations of the energy density around its mean value will also be considered, and some recent results on a probability distribution for the energy density in two dimensional spacetime are summarized.
\end{abstract}

\section{Introduction}

Although the local energy density for the electromagnetic and other known fields is positive at the classical level, this need not be the case at the quantum level. When defining the expectation value of the stress tensor in quantum field theory, a subtraction is needed, after which a negative value can result. In the case of empty Minkowski spacetime, we take the vacuum state as the zero of energy density. Negative values for the mean energy density are examples of sub-vacuum phenomena, where the effects of vacuum fluctuations have effectively been suppressed. We will review some examples of this effect in the Casimir effect and in non-classical quantum states, and also review the quantum inequalities which limit sub-vacuum phenomena. In Sect. 5, some proposed laboratory experiments to detect sub-vacuum effects will be discussed. Finally we turn to the topic of stress tensor fluctuations, whereby negative energy can arise by a quantum fluctuation even when the expectation value of the energy is positive or zero. Some recent results on the probability distribution for such fluctuations will be discussed. 


\section{Negative Energy Density in the Casimir Effect}

Simply from Casimir's result for the force per unit area ${ }^{1}$ between a pair of parallel perfectly reflecting plates, one can construct the entire stress tensor, using conservation, tracelessness and symmetry arguments. ${ }^{2,3}$ The result, in units where $\hbar=c=1$, is

$$
T_{\mu \nu}=\left(\begin{array}{cccc}
T_{00} & 0 & 0 & 0 \\
0 & T_{x x} & 0 & 0 \\
0 & 0 & T_{y y} & 0 \\
0 & 0 & 0 & T_{z z}
\end{array}\right)=\frac{\pi^{2}}{720 a^{4}}\left(\begin{array}{cccc}
-1 & 0 & 0 & 0 \\
0 & 1 & 0 & 0 \\
0 & 0 & 1 & 0 \\
0 & 0 & 0 & -3
\end{array}\right)
$$

where the plates are separated by a distance $a$ in the $z$-direction. Thus there is a constant negative energy density between the plates.

The case of plates of finite reflectivity is more complicated, and the local stress tensor can no longer be recovered simply from knowledge of the force per unit area. The reason for this is that symmetry under Lorentz boosts parallel to the plates, a key ingredient in the argument leading to Eq. (1), no longer holds. Helfer and $\mathrm{Lang}^{4}$ have noted that now there could be a positive self-energy density associated with each plate, even when the plates are widely separated. In this case, an attractive force is no guarantee of negative energy density. Consider for example the case of classical electrostatics, where opposite charges attract, but the local energy density, proportional to the squared electric field, in non-negative. In the case of plates described by a plasma model dielectric function, the local energy density between the plates was calculated in Ref. 5, with the results illustrated in Fig. 1.

The basic result is that the local energy density at the center of the region between the plates will become negative when the reflectivity of the plates is sufficiently large, specifically when $\omega_{p} a>100$, where $\omega_{p}$ is the plasma frequency. Thus local negative energy density is possible in the Casimir effect, but is not inevitable.

\section{Negative Energy from Quantum Coherence Effects}

Another way to create local negative energy densities is with non-classical states of the quantized electromagnetic field, or indeed any quantum field. It was proven many years ago by Epstein et al ${ }^{6}$ that all quantum field theories contain quantum states in which the local energy density at a given point may be made negative. In fact, it can be arbitrarily negative. ${ }^{7}$ Explicit examples of this phenomenon are rather easy to construct, and include the moving mirror models of Fulling and Davies ${ }^{8}$ and the squeezed 


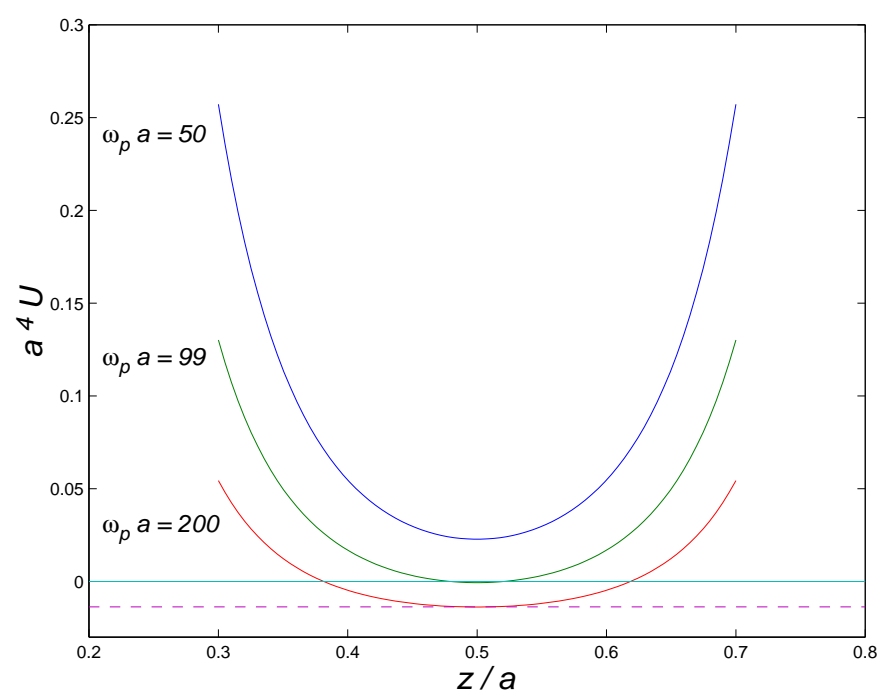

Fig. 1. The energy density in the vacuum region between two dielectric half-spaces is illustrated for three values of the parameter $\omega_{p} a$. The dashed horizontal line is the energy density for the perfectly conducting limit.

vacuum states. ${ }^{9}$ The energy density in several non-classical states, including squeezed vacua, is discussed, for example, in Refs. 10,11.

In the case of a single monochromatic mode, the energy density will be oscillatory, with both negative and positive energy density intervals, but with the time-averaged energy being positive. By making the frequency of the mode arbitrarily high, one can make the peak values of both the negative and positive energy densities arbitrarily large, but the duration of the period of negative energy will become arbitrarily short. As we will see in Sect. 4, this is a very general feature of negative energy from quantum coherence effects.

Unrestricted negative energy would have dramatic and disturbing consequences, including violations of the second law of thermodynamics, ${ }^{12}$ formation of naked singularities in general relativity, ${ }^{13,14}$ traversable wormholes, ${ }^{15,16}$ and faster than light travel. ${ }^{17}$ The later two possibilities could allow the creation of a time machine, with all of the logical problems inherent in time travel. 


\section{Quantum Inequalities}

Unrestricted negative energy would cause serious problems for physics as it is presently formulated. This leads to the suspicion that the laws of physics do place restrictions on negative energy and related effects. This is indeed the case. In the case of free fields in Minkowski spacetime, it is possible to prove "quantum inequalities" which greatly constrain the magnitude and duration of negative energy fluxes or periods of negative energy density. The first versions ${ }^{12,18}$ of the quantum inequalities were limits on the negative energy fluxes, which showed that the duration of such a flux has an inverse relation to its magnitude. This limit is sufficient to show that macroscopic violations of the second law are not possible. Later authors ${ }^{19-23}$ proved quantum inequalities for the expectation value of the energy density in arbitrary quantum states. Let

$$
\rho(t)=\left\langle T_{t t}(t)\right\rangle
$$

be the expectation value of the energy density operator in an arbitrary state, evaluated on the worldline of an inertial observer at time $t$. Further, let $g(t, \tau)$ be a sampling function in $t$ with a characteristic width of $\tau$. The quantum inequalities for a massless field take the general form

$$
\int \rho(t) g(t, \tau) d t \geq-\frac{C}{\tau^{d}}
$$

where $d$ is the spacetime dimension, and $C$ is a positive constant. The basic physical content of Eq. (3) is that a observer who sees negative energy lasting for a time of order $\tau$ will measure the magnitude of this negative energy density to be bounded by about $C / \tau^{d}$.

In the case of a massless scalar field in two-dimensional spacetime, $d=2$, Flanagan $^{21}$ has obtained the optimum bound to be given by

$$
C=\frac{\tau^{2}}{24 \pi} \int_{-\infty}^{\infty} d t \frac{\dot{g}^{2}}{g}
$$

where $\dot{g}=\partial g / \partial t$. For example, for the case of a Gaussian sampling function, $g(t, \tau)=(\sqrt{\pi} \tau)^{-1} \mathrm{e}^{-t^{2} / \tau^{2}}$, one finds $C=1 /(12 \pi)$. This bound will play a key role in the results on energy density fluctuations to be discussed in Sect. 6. Flanagan's bound, Eq. (4), is optimal in the sense that one can construct a quantum state for which this relation is an equality, thus proving that there cannot be a more restrictive bound for arbitrary states. In four dimensional spacetime, the optimal bound is not known, but Fewster and Eveson $^{22}$ have proven an inequality for a general sampling function. In this 
case, typical values of $C$ are of order $10^{-3}$ for functions such Lorentzians or Gaussians.

It may be shown ${ }^{12-14,20,25,26}$ that these inequalities, and their curved spacetime analogs, greatly restrict the effects of negative energy, making macroscopic violations of the second law or of cosmic censorship unlikely, and preventing the construction of macroscopic wormholes or warpdrives.

\section{Possible Experiments to Detect Negative Energy?}

Although negative energy and related sub-vacuum effects are constrained by quantum inequalities, this does not mean that they are unobservable in principle. Unfortunately, the gravitational effects of negative energy are extremely small. However, there is still a possibility of a laboratory experiment using non-gravitational effects. The role of quantum inequalities in quantum optics has been discussed in Refs. 27,28.

An early attempt to devise an experiment was made in Ref. 29. These authors considered a spin system in a magnetic field interacting with the quantized electromagnetic field in a non-classical state, such as a squeezed vacuum, for a single plane wave mode. Normally, photons will flip spins and cause a decrease in the magnetization of the system. However, in a non-classical photon state, the instantaneous magnetization can actually increase above its value in the vacuum. In the model of Ref. 29, this increase occurs during the interval when the expectation value of energy density of the quantized electromagnetic field is negative. One can interpret this result as follows: quantum vacuum fluctuations cause some de-alignment of the spins, compared to what would occur in a world without vacuum fluctuations. One cannot turn off these fluctuations, but they can be momentarily suppressed, resulting in "repolarization". This effect seems too small for a realistic experiment. It should also be noted that it is really measuring the mean squared magnetic field $\left\langle B^{2}\right\rangle$ in the non-classical state, rather than the energy density. It is only in the special case of a plane wave mode, where $\left\langle B^{2}\right\rangle=\left\langle E^{2}\right\rangle$, that this effect is a measure of negative energy density.

Another proposal to measure sub-vacuum effects was made in Ref. 30, where the effect of squeezed states of the photon field on electron interference was discussed. Normally, the scattering of photons by the electrons will lead to dephasing, decreasing the contrast of the interference pattern. However, when the photons are in a squeezed vacuum state, and the interference pattern is formed only from electrons which pass through the interferometer at selected times in the cycle of the excited mode, then the contrast can increase compared to the vacuum case. This effect of "recoher- 
ence" is similar to the repolarization effect for spins. Vacuum fluctuations of the electromagnetic field cause fluctuations of the Aharonov-Bohm phase of the electrons, which in turn lead to a loss of contrast, compared to what would be seen in a world without vacuum fluctuations. Again, the best that we can do is to momentarily suppress these fluctuations, and cause a small increase in contrast. This effect can be expressed as a change in a double surface integral of $\left\langle F_{\mu \nu}(x) F_{\alpha \beta}\left(x^{\prime}\right)\right\rangle$, the electromagnetic field strength correlation function, with the integration taken over a surface bounded by the electron paths. This is not a local quantity like the energy density, but suppression of the Aharonov-Bohm phase fluctuation is a sub-vacuum effect, just as is negative energy density. The effect of recoherence is in principle observable, but probably not with current technology.

Another potentially observable effect of sub-vacuum phenomena arises from the spontaneous decay rates of atoms. It is well known that vacuum fluctuations are essential for spontaneous decay, because atomic energy levels would be eigenstates of the Hamiltonian, and hence stable, were it not for the coupling to the quantized electromagnetic field. It was recently proposed in Ref. 31 that non-classical states of the photon field might lead to observable suppression of atomic decay rates. The proposed experiment involves sending a beam of atoms in an excited state through a cavity, in which one mode is excited in a non-classical state, such as a squeezed vacuum. On average, the effect of the excited state will be to increase the decay rate, as would be expected from stimulated emission effects. However, if the atoms pass through the cavity at certain times in the cycle of the excited mode, then the decay probability during the transit period can be reduced compared to the case when the electromagnetic field in the cavity is in the vacuum state. As in the effects discussed in the previous two paragraphs, this can be interpreted as suppression of the usual vacuum fluctuation effects. Under certain conditions, this effect can serve to measure $\left\langle E^{2}\right\rangle$, the shift in mean squared electric field due to the non-classical state (but not including Casimir effects due solely to the cavity). In the case where the frequency of the cavity field is near the atomic transition frequency, the transit time is small compared to the associated period, and the mode function is approximately constant along the atom's path, then the decay probability is

$$
\frac{P}{P(0)}=1+\frac{1}{f^{2}\left(\mathbf{x}_{\mathbf{0}}\right)}\left\langle E^{2}\left(\mathbf{x}_{\mathbf{0}}, t\right)\right\rangle .
$$

Here $f^{2}\left(\mathbf{x}_{\mathbf{0}}\right)$ is the squared mode function for the excited mode, evaluated at a point $\mathbf{x}_{\mathbf{0}}$ on the atom's path, $P$ is the decay probability for the non- 
classical state, and $P(0)$ is that for the vacuum state. When $\left\langle E^{2}\left(\mathbf{x}_{\mathbf{0}}, t\right)\right\rangle<0$, then $P<P(0)$, and the decay rate has been suppressed. In Ref. 31, a quantum inequality is proven which states that

$$
\left\langle E^{2}\left(\mathbf{x}_{\mathbf{0}}, t\right)\right\rangle \geq-f^{2}\left(\mathbf{x}_{\mathbf{0}}\right),
$$

thus guaranteeing that $P \geq 0$, as required. However, a state which comes close to saturating the quantum inequality bound will lead to a significant fractional decrease in decay probability when the atom is in the cavity. Note that this is quite different from the more familiar suppression of atomic decay rates in cavities compared to empty space, which can be interpreted in terms of a lack of available modes in to which to atom can decay. The decay probability during transit is small to begin with, of order $10^{-8}$ in some examples treated in Ref. 31, so a large number of atom needs to be used to produce a statistically significant result. However, a realistic experiment might be feasible, and is currently under study.

\section{Quantum Stress Tensor Fluctuations}

So far, we have been discussing situations where the expectation value of the energy density can be negative. However, there is another sense in which negative energy density can arise in quantum field theory. This is when quantum fluctuations momentarily create a region of negative energy. This can occur even when the expectation value is non-negative. A simple example is the Minkowski vacuum state, where $\left\langle T_{\mu \nu}\right\rangle=0$, but the state is not an eigenstate of $T_{\mu \nu}$. This means that there must be both positive and negative fluctuations and an associated probability distribution function. To find this, we need the probability distribution for a stress tensor operator averaged over a sampling function in time, or spacetime.

In general, this is still an unsolved problem. However, it has recently been solved for the case of two-dimensional conformal field theory with a Gaussian average in time. ${ }^{32}$ Here we simply quote the results, which will be derived in Ref. 32, and discuss some of their physical implications. Let $T_{t t}$ be the energy density operator, and define the averaged energy density by

$$
u=\frac{1}{\sqrt{\pi} \tau} \int_{-\infty}^{\infty} T_{t t}(t, x) \mathrm{e}^{-t^{2} / \tau^{2}} d t .
$$

The associated probability distribution is a Gamma distribution given by

$$
P(x)=\frac{\pi^{c / 12}}{\Gamma(c / 12)}\left(x+x_{0}\right)^{c / 12-1} \mathrm{e}^{-\pi\left(x+x_{0}\right)}, \quad x>-x_{0},
$$


and $P(x)=0$ for $x<-x_{0}$. Here $x=u \tau^{2}, c$ is the central charge, and $-x_{0} / \tau^{2}$ is the quantum inequality bound on expectation values of $T_{t t}$ in arbitrary quantum states. The probability distribution is plotted in Fig. 2 for the case of a free massless scalar field in two-dimensional Minkowski spacetime, for which $c=1$.

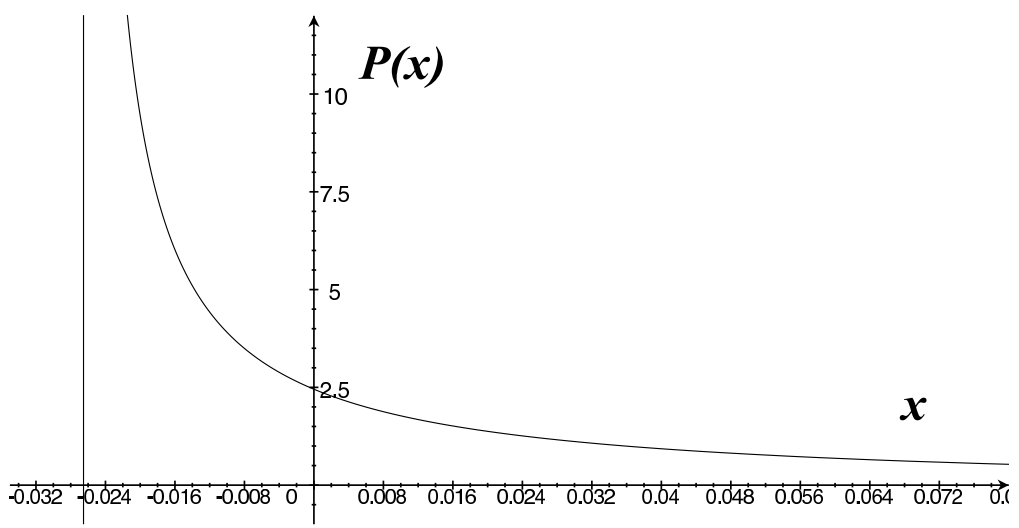

Fig. 2. The probability distribution $P(x)$ for the smeared energy density of a massless scalar field in two-dimensional Minkowski spacetime is plotted. Here $x=u \tau^{2}$, where $u$ is the energy density operator averaged in time with a Gaussian function of width $\tau$. The lower limit of $P(x)$ occurs at $x=-x_{0}=-1 /(12 \pi)$, illustrated by the vertical line.

The lower cutoff at the quantum inequality bound is expected to be a general feature of the probability distribution for vacuum fluctuations of an averaged energy density. It is of interest to note that 0.84 of the area of the graph in Fig. 2 lies to the left of the origin. This means that a measurement of the averaged energy density will find a negative result $84 \%$ of the time. However, when a positive value is found, it is typically larger in magnitude.

The probability distribution for the energy density in four-dimensional theories has not yet been found, but is of considerable interest. One application is to inflationary cosmology, where quantum stress tensor fluctuations might contribute a potentially observable component to the cosmological density fluctuations. ${ }^{33}$ This component would be non-Gaussian in a way which is associated with the skewness of the quantum stress tensor probability distribution. A distinct, more exotic, application to cosmology arises in models which employ anthropic reasoning to compute probabilities of various observables. These models require a counting of observers, usually assumed to be beings like ourselves in the sense of having arisen from bi- 
ological evolution on earth-like planets. However, another possibility are "Boltzmann brains" which have nucleated from the vacuum in deSitter or even Minkowski spacetime. Quantum energy fluctuations can sometimes produce large concentrations of energy, which very occasionally might be capable of conscious thought. The probability per unit volume of this is very tiny, but the available volume is vastly larger than that for biological systems. If "Boltzmann brains" are the more prevalent type of observer, it would greatly complicate attempts at anthropic prediction. (For further discussion and references, see, for example Refs. 34,35.) The key to studying this question is in the details of the long positive tail of the probability distribution, which can tell us how likely the appearance of a "Boltzmann brain" in a given region might be.

\section{Summary}

We have seen that quantum field theory allows local negative energy and other sub-vacuum effects. These effects are strongly restricted by quantum inequalities, but are nonetheless potentially observable. We have reviewed some proposals to measure these effects in laboratory experiments, the most promising of which involves changes in the decay rates of atoms, and could conceivably lead to measurements of negative mean squared electric fields. We have also discussed some new results on the probability distribution for vacuum stress tensor fluctuations. This distribution has a lower cutoff at the quantum inequality bound on the expectation value in an arbitrary state, but has a tail in the positive direction. Thus a typical fluctuation in the local energy density is negative but bounded below, while rare but extremely large positive fluctuations are possible. Stress tensor fluctuations effects could be important in inflationary cosmology.

\section{Acknowledgments}

I would like to thank Chris Fewster and Tom Roman for valuable discussions. This research was supported in part by the US National Science Foundation under Grant No. PHY-0855360.

\section{References}

1. H. B. G. Casimir, Proc. Kon. Ned. Akad. Wet. 51, 793 (1948).

2. L. S. Brown, G. J. Maclay, Phys. Rev. 184, 1272 (1969).

3. B.S. DeWitt, Phys. Rep. 19C, 295 (1975).

4. A. D. Helfer and A. S. Lang, J. Phys. A: Math. Gen. 32, 1937 (1999). 
5. V. Sopova and L.H. Ford, Phys. Rev. D 66, 045026 (2002), quant-ph/0204125.

6. H. Epstein, V. Glaser, and A. Jaffe, Nuovo Cim. 36, 1016 (1965).

7. C.J. Fewster, "Energy inequalities in quantum field theory", in Proceedings of the XIVth International Congress on Mathematical Physics, edited by J.C. Zambrini (World Scientific, Singapore, 2005), math-ph/0501073

8. S.A. Fulling and P.C.W. Davies, Proc. R. Soc. London A348, 393 (1976); P.C.W. Davies and S.A. Fulling, Proc. R. Soc. London A356, 237 (1977).

9. C. M. Caves, Phys. Rev. D 23, 1693 (1981).

10. A. Borde, L.H. Ford, and T.A. Roman, Phys. Rev. D 65, 084002 (2002), qr-qc/0109061.

11. L.H. Ford and T.A. Roman, Phys. Rev. D 77, 045018 (2008), arXiv:0705.3003.

12. L. H. Ford, Proc. Roy. Soc. Lond. A364, 227 (1978).

13. L.H. Ford and T.A. Roman, Phys. Rev. D 41, 3662 (1990).

14. L.H. Ford and T.A. Roman, Phys. Rev. D 46,1328 (1992).

15. M. Morris, K. Thorne, and U. Yurtsever, Phys. Rev. Lett. 61, 1446 (1988).

16. M. Visser, Lorentzian Wormholes; From Einstein to Hawking, (AIP, Woodburry, N. Y., 1995).

17. M. Alcubierre, Class. Quantum Grav. 11, L73 (1994).

18. L. H. Ford, Phys. Rev. D43, 3972 (1991).

19. L.H. Ford and T.A. Roman, Phys. Rev. D 51, 4277 (1995), gr-qc/9410043.

20. L.H. Ford and T.A. Roman, Phys. Rev. D 55, 2082 (1997), gr-qc/9607003.

21. E.E. Flanagan, Phys. Rev. D, 56, 4922 (1997), gr-qc/9706006.

22. C.J. Fewster and S.P. Eveson, Phys. Rev. D 58, 084010 (1998), grqc/9805024.

23. C.J. Fewster, Class. Quantum Grav. 17, 1897 (2000), gr-qc/9910060.

24. L.H. Ford and T.A. Roman, Phys. Rev. D 53, 5496 (1996), gr-qc/9510071.

25. M.J. Pfenning and L.H. Ford, Class. Quantum Grav., 14, 1743 (1997), grqc/9702026.

26. T.A. Roman, "Some Thoughts on Energy Conditions and Wormholes", in Proceedings of the Tenth Marcel Grossmann Meeting on General Relativity, edited by S.P. Bergliaffa and M. Novello, (World Scientific, Singapore, 2006), gr-qc/0409090.

27. P. Marecki, Phys. Rev. A 66, 053801 (2002), quant-ph/0203027.

28. P. Marecki and N. Szpak, Ann. Phys. (Leipzig) 14, 428 (2005), quant$\mathrm{ph} / 0407186$.

29. L.H. Ford, P.G. Grove, and A.C. Ottewill, Phys. Rev. D 46, 4566 (1992).

30. J.T. Hsiang and L.H. Ford, Phys. Rev. D 78, 065012 (2008), rXiv:0806.1512.

31. L.H. Ford and T.A. Roman, arXiv:0907.1638.

32. C.J. Fewster, L.H. Ford, and T.A. Roman, manuscript in preparation.

33. C.-H. Wu, K.-W. Ng, and L.H. Ford, Phys. Rev. D 75, 103502 (2007), grqc/0608002.

34. G. Garriga and A. Vilenkin, Phys. Rev. D 77, 043526 (2008), arXiv:0711.2559.

35. A. De Simone, A.H. Guth, A. Linde, M. Noorbala, M.P. Salem, and A. Vilenkin, arXiv:0808.3778. 